\title{
Acupuncture for Posttraumatic Stress Disorder: A Systematic Review of Randomized Controlled Trials and Prospective Clinical Trials
}

\author{
Young-Dae Kim, ${ }^{1}$ In Heo, ${ }^{1}$ Byung-Cheul Shin, ${ }^{1,2}$ Cindy Crawford, ${ }^{3}$ \\ Hyung-Won Kang, ${ }^{4}$ and Jung-Hwa Lim $^{5}$ \\ ${ }^{1}$ School of Korean Medicine, Pusan National University, Yangsan 626-870, Republic of Korea \\ ${ }^{2}$ Division of Clinical Medicine, School of Korean Medicine, Pusan National University, Yangsan 626-870, Republic of Korea \\ ${ }^{3}$ Samueli Institute, 1737 King Street, Suite 600, Alexandria, VA 22314, USA \\ ${ }^{4}$ Department of Neuropsychiatry \& Herbal Resources, Professional Graduate School of Korean Medicine, \\ Won-Kwang University, Iksan 570-749, Republic of Korea \\ ${ }^{5}$ Department of Neuropsychiatry, Korean Medical Hospital, Pusan National University, Yangsan 626-789, Republic of Korea
}

Correspondence should be addressed to Byung-Cheul Shin; drshinbc@gmail.com

Received 9 August 2012; Accepted 15 October 2012

Academic Editor: Hans-Christian Deter

Copyright (C) 2013 Young-Dae Kim et al. This is an open access article distributed under the Creative Commons Attribution License, which permits unrestricted use, distribution, and reproduction in any medium, provided the original work is properly cited.

To evaluate the current evidence for effectiveness of acupuncture for posttraumatic stress disorder (PTSD) in the form of a systematic review, a systematic literature search was conducted in 23 electronic databases. Grey literature was also searched. The key search terms were "acupuncture" and "PTSD." No language restrictions were imposed. We included all randomized or prospective clinical trials that evaluated acupuncture and its variants against a waitlist, sham acupuncture, conventional therapy control for PTSD, or without control. Four randomized controlled trials (RCTs) and 2 uncontrolled clinical trials (UCTs) out of 136 articles in total were systematically reviewed. One high-quality RCT reported that acupuncture was superior to waitlist control and therapeutic effects of acupuncture and cognitive-behavioral therapy (CBT) were similar based on the effect sizes. One RCT showed no statistical difference between acupuncture and selective serotonin reuptake inhibitors (SSRIs). One RCT reported a favorable effect of acupoint stimulation plus CBT against CBT alone. A meta-analysis of acupuncture plus moxibustion versus SSRI favored acupuncture plus moxibustion in three outcomes. This systematic review and meta-analysis suggest that the evidence of effectiveness of acupuncture for PTSD is encouraging but not cogent. Further qualified trials are needed to confirm whether acupuncture is effective for PTSD.

\section{Introduction}

Posttraumatic stress disorder (PTSD) develops following a stressful event or situation of an exceptionally threatening or catastrophic nature, which is likely to cause pervasive distress [1]. PTSD is classified as an anxiety disorder and is typically defined by the coexistence of 3 clusters of symptoms, namely, reexperiencing, marked avoidance, and hyperarousal [2]. The prevalence rates of PTSD have been reported as 6$25 \%$ [3], and approximately $25-30 \%$ of people experiencing a traumatic event may go on to develop PTSD [4].

Current first-line PTSD therapies include trauma-focused cognitive behavioral therapy (CBT), stress inoculation training, and pharmacotherapies [5]. Complementary and alternative medicine (CAM) interventions include a range of therapies that are not considered standard to the practice of medicine in the USA. CAM therapies are widely used by mental health consumers, including veterans, and numerous stakeholders have expressed strong interest in fostering the evidence base for these approaches in PTSD [6]. In addition, approximately $21 \%$ of CAM users met diagnostic criteria for at least one problematic mental disorder, according to one study [7].

Acupuncture is commonly recognized worldwide as a mainstream CAM therapy. Acupuncture is the practice of inserting a needle or needles into certain points in the 
body, known as meridian acupuncture points, for therapeutic or preventive purposes [8]. Numerous studies have shown that acupuncture is well tolerated by patients, safe, and cost effective compared to routine care [9].

Additionally, acupuncture is widely used in mental disorders such as anxiety disorders [10], dementia [11], eating disorders [12], schizophrenia [13], sleep disorders [14], and substance-related disorders $[15,16]$. Electroacupuncture is effective in rat models of stress and thus might be a useful adjunct therapy in stress-related anxiety disorders [17]. Acupuncture has positive effects in PTSD patients, although the evidence is still lacking as to its true efficacy for this condition [18].

There have been two reviews published on acupuncture or its variants for PTSD $[19,20]$. David Feinstein reviewed 2 randomized controlled trials (RCTs) and 6 outcome studies which tested whether brief psychological exposure with acupoint tapping was effective for PTSD or not and its conclusion was not confirmative [19]. Also Michael Hollifield reviewed acupuncture for PTSD referring one published and one unpublished clinical trial and suggested further definitive research is needed because of lack of well-conducted RCTs [20]. However, there has been no systematic review published to date fully summarizing the current total evidence about the quality and effectiveness of acupuncture for PTSD. For this reason, we conducted a systematic review of RCTs and prospective clinical trials to assess critically whether acupuncture improves the symptoms of PTSD and to make recommendations for future research based on gap areas identified in this review.

\section{Methods}

2.1. Data Sources and Search Strategy. Following the COSI model [21], we searched the following electronic databases over time periods from their inception to July 2012: Cochrane Database of Systematic Reviews, the Cochrane Central Register of Controlled Trials (CENTRAL), MEDLINE through PubMed, EMBASE, Allied and Complementary Medicine Database (AMED), CINAHL, Pilots, Google, Korean databases (which include DBpia, Korea Institute of Science and Technology Information (KISTI), KoreaMed, Korean traditional knowledge portal, OASIS, RISS, the National Assembly Library, and The National Library of Korea), Chinese databases (which include China Academic Journal, http://www.cqvip.com and WANFANG DATA), and a Japanese database (Japan Science and Technology Information Aggregator Electronic). We also searched the grey literature; unpublished trials were searched via the Register of the Controlled Trials databases ( http://www.controlled-trials.com and http://www.clinicaltrials.gov), and we communicated with identified experts in the field of acupuncture and PTSD, searched our departmental files, and pearled the references of all included articles for other relevant articles perhaps not picked up through other methods of searching.

The key search terms were "(acupuncture OR acup*) AND (stress disorders, post-Traumatic OR posttraumatic stress disorder OR posttraumatic stress disorder OR PTSD)." $\mathrm{MeSH}$ strategy was applied to ensure the most powerful search where applicable. Search strategies were adjusted for each of the databases. Personal contacts were made with the original authors of the searched studies to identify any potential missing data from the publications.

2.2. Study Selection. Two psychiatrists (J. H. Lim and H. W. Kang) actively participated in the study selection process based on clinical expertise, and two experienced researchers (B. C. Shin, C. Cindy) monitored the whole process of systematic review. All reviewers were fully trained in the systematic review process executed.

2.2.1. Types of Studies. The review was not restricted by study design, however, study should be prospective clinical trials. We included RCTs and nonrandomized controlled trials that compared acupuncture or its variants with a control or control groups. We also included uncontrolled clinical trials (UCTs) of acupuncture for PTSD to give our research question a more solid ground or to make recommendations for future research. However, we separately analyzed RCTs and others, and interpreted more weighted on RCTs because of research quality following the validity of evidence. No restrictions were imposed on studies with regard to blinding, languages, or year published.

2.2.2. Types of Participants. We selected all studies including patients with PTSD diagnosed by any set of criteria, DSM-IV or ICD-10, regardless of gender, age, nationality, or outpatient therapy or inpatient therapy.

2.2.3. Types of Interventions/Controls. Clinical trials investigating any type of needling acupuncture, specifically classical acupuncture, electroacupuncture, auricular acupuncture were included. We also included trials that included acupuncture as a more complex intervention, that is, acupuncture plus another intervention if the comparison group was that other intervention. We included trials using control groups with no treatment, sham/placebo acupuncture, and conventional treatments for PTSD patients. We excluded laser acupuncture and acupoint stimulation such as acupressure, moxibustion, tapping, and so forth because of the lack of needling. We excluded trials with controls that acted as "healthy participants."

2.2.4. Types of Outcome Measures. The most recent guideline for treatment of PTSD [5] includes the following major outcomes: 1st: "reduction in severity of PTSD symptoms"; 2nd: "prevention/reduction of trauma-related comorbid conditions"; 3rd: "patient adherence to treatment plan"; 4th:"response to treatment"; 5th: "social, occupational, adaptive, and interpersonal functioning"; 6th: "quality of life" and 7th: "rate of relapse." The main outcome measures were any relevant PTSD scales as clinician-administered PTSD scale (CAPS), depression scale, and anxiety scale. Other scales as related to impairment, proportion of patients recovered were extracted following predefined protocol. 
2.3. Screening, Data Extraction, and Quality Assessment. After screening titles and abstracts retrieved through our search, we excluded all articles that did not match our inclusion/exclusion criteria according to the predefined eligibility criteria mentioned above. Then, expected inclusions were carefully read in full text, and final inclusion was decided by two independent reviewers (Y. D. Kim, I. Heo) by matching method. If studies were written in languages incomprehensible for the reviewers, all articles not written in native language were translated by colleagues. Then we first classified these by the eligibility criteria. If there was a need for full text review, we evaluated these after translation. Data were extracted independently based on predefined characteristics to describe each study (refer to Table 1) by the two reviewers. All disagreements were resolved by discussion and consensus, or by the first author. The Cochrane risk of bias for assessing the quality of included RCTs [22], the CONSORT 2010 checklist for reporting quality of RCTs [23] and the revised standards for reporting interventions in clinical trials of acupuncture (STRICTA) guideline for reporting quality of acupuncture trials [24] were used to evaluate the methodological quality of the included publications. All reviewers were fully trained in the quality assessment and data extraction methodology.

2.4. Data Synthesis and Statistics. Two authors (Y. D. Kim, B. C. Shin) calculated effect estimates (effect size: ES) to summarize the effects of acupuncture on each outcome by recalculation for mean and standard deviation (SD) because all original data were continuous ones. The standardized mean difference (SMD) and 95\% confidence interval (CI) on each outcome measurement were calculated using Cochrane Collaboration software (Review Manager (RevMan) Version 5.1.7 for Windows. Copenhagen: The Nordic Cochrane Centre). For meta-analysis, we pooled data across studies using weighted mean difference (WMD) because same measurement was used. Random effect model was used because clinical heterogeneities were expected across the studies. To assess the heterogeneity among the trials, Chi-square test and the Higgins $I^{2}$ test were used.

\section{Results}

3.1. Study Description. The searches retrieved 136 potentially relevant articles. After screening the titles and abstracts, we excluded 120 studies (Figure 1). 16 articles were read in full and evaluated. Subsequently, 5 studies were excluded because 1 was a controlled trial but the control group members were healthy subjects [30], 1 was active status not recruiting [31], 1 was recruiting status [32], and 2 were completed but with the results not published $[33,34]$. Finally 9 RCTs and 2 UCTs were identified. Of 9 RCTs published, Zhang et al. RCT [25] was split or duplicated published with same data $[25,35-$ 39]. So we included only 1 RCT with full data [25] from the 6 RCTs [25, 35-39]. Consequently, 4 RCTs [18, 25-27] and 2 UCTs $[28,29]$ met our inclusion criteria. Figure 1 sums up the search results based on a four-phase flow diagram in Preferred Reporting Items for Systematic Reviews and MetaAnalyses (PRISMA) statement format [40]. The key data are summarized in Table 1. One RCT originated from the USA [18], while all the others were from China [25-29]. All RCTs adopted a parallel-group design. Two of them were twoparallel-arm group designs [26, 27], one was a three-parallelarm group design [18], and one was a four-parallel-arm group design [25]. Two RCTs [18, 25] were based on a sample size calculation, whereas the other two RCTs $[26,27]$ did not report this.

The four RCTs evaluated 543 PTSD patients (mean sample size per arm: 49). The duration of treatment was 1 to 12 weeks. A table showing baseline clinical characteristics for each group was reported in only one RCT [18].

3.2. Interventions. One RCT compared needle acupuncture to cognitive-behavioral therapy (CBT) and a waitlist control [18], and another used electroacupuncture only or with moxibustion or with auricular acupuncture versus oral selective serotonin reuptake inhibitors (SSRIs) [25]. One RCT tested electroacupuncture plus moxibustion versus oral SSRI [26], and one RCT compared acupoint stimulation plus CBT to CBT alone [27]. One UCT [29] used just acupuncture, the other UCT [28] used electroacupuncture plus auricular acupuncture and moxibustion. 1 RCT [18] and 1 UCT [29] used manual stimulation without electrical stimulation, and the other 3 RCTs [25-27] and 1UCT [28] used electrical stimulation.

\subsection{Outcomes}

3.3.1. Acupuncture versus CBT/Acupuncture versus Waitlist Control/CBT versus Waitlist Control. One high-quality RCT evaluated the effect of acupuncture against CBT and a waitlist control [18]. No statistical difference was found between acupuncture and CBT. But, acupuncture treatment was statistically superior to waitlist control on four outcome measures; posttraumatic symptom scale-self report (PSS-SR) (ES, $-0.98 ; P=0.001)$, Depression: self-rated Hopkins symptom checklist-25 (HSCL-25) (ES, $-0.68 ; P=0.02$ ), Anxiety: HSCL-25 (ES, $-0.91 ; P=0.003)$, and Impairment: Sheehan Disability Inventory (SDI) (ES, $-0.64 ; P=0.03$, Table 1). The CBT was also statistically superior to waitlist control on four outcome measures; PSS-SR (ES, $-0.85 ; P=$ $0.004)$, Depression: HSCL-25 (ES, $-0.80 ; P=0.008$ ), Anxiety: HSCL-25 (ES, $-0.79 ; P=0.008$ ), Impairment (ES, -0.64 ; $P=0.03)$. The therapeutic effects of acupuncture and CBT were similar on the ESs [41] (Table 1).

3.3.2. Acupuncture versus Oral SSRI. One RCT evaluated the effect of electroacupuncture versus oral SSRI [25]. No statistical difference was found between two groups.

3.3.3. Acupuncture Plus CBT versus CBT Alone. One RCT assessed the effect of acupoint stimulation plus CBT in comparison to CBT alone [27]. Recalculation of the mean difference (MD) revealed a favorable effect of acupoint stimulation plus CBT in terms of IES-R (ES, $-1.56 ; P<$ 0.00001 ) and the self-compiled questionnaire (ES, $-0.59 ; P=$ 0.01) (Table 1). 


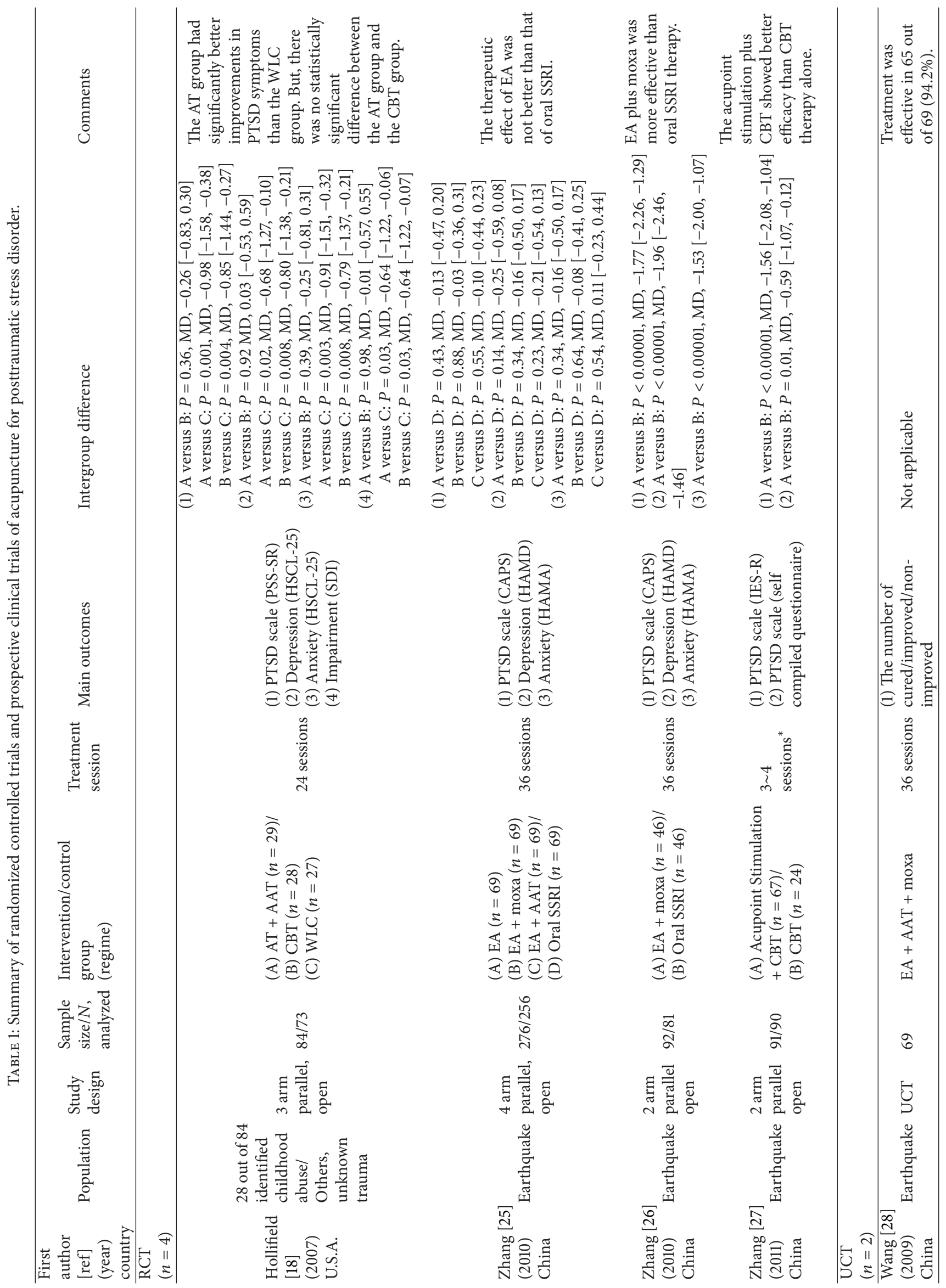




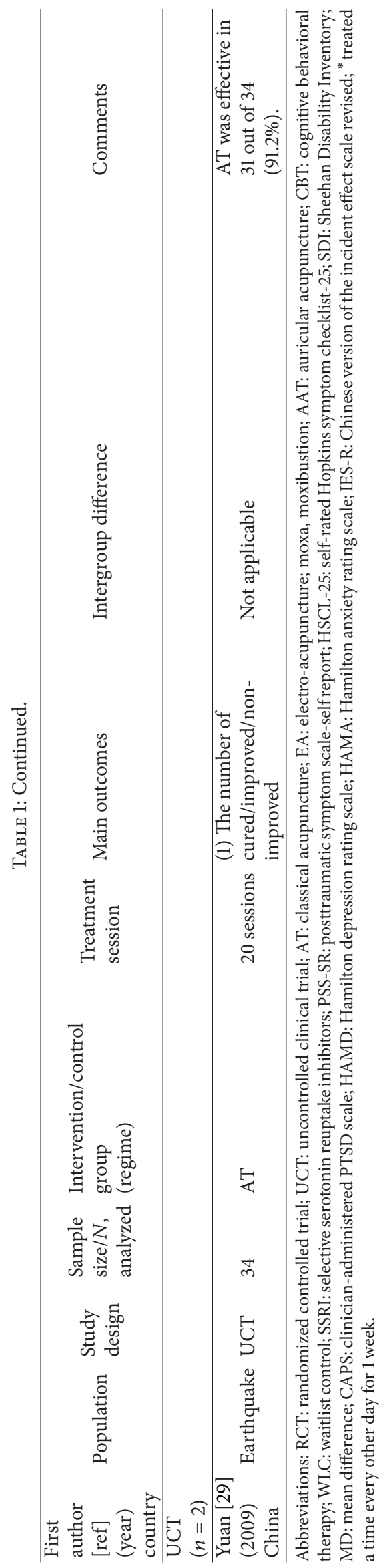




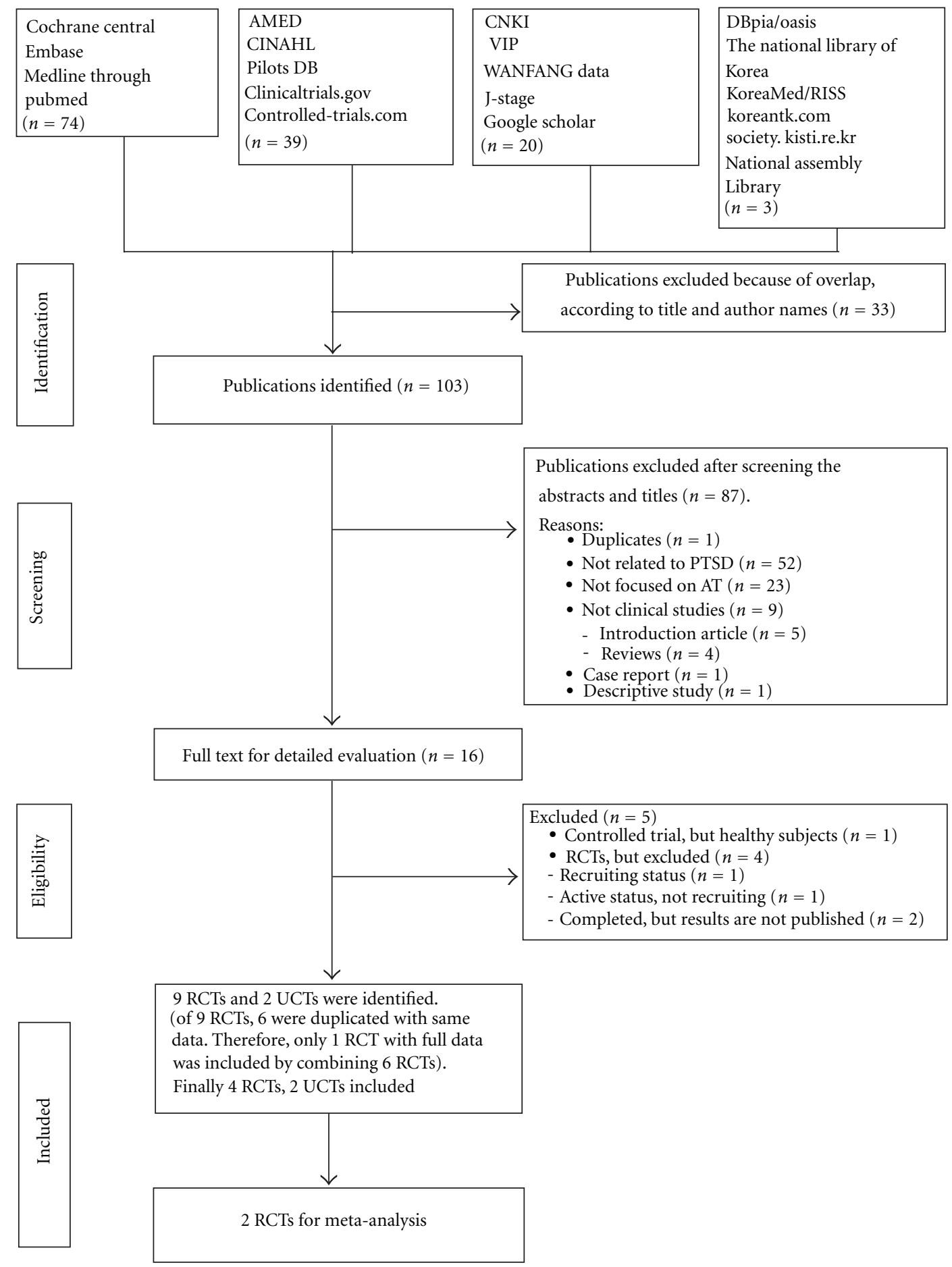

FIGURE 1: Flow chart of the trial selection process. PTSD: posttraumatic stress disorder; RCT: randomized controlled trial; UCT: uncontrolled clinical trial; AT: acupuncture.

3.3.4. Acupuncture Plus Moxibustion versus Oral SSRI. Two RCTs assessed the effects of electroacupuncture plus moxibustion against oral SSRI $[25,26]$. One RCT reported no statistical difference between the two groups [25]. However, the other RCT showed that electroacupuncture plus moxibustion was statistically superior to oral SSRI on outcome clinicianadministered PTSD scale (CAPS) (ES, $-1.77 ; P<0.00001)$, depression (ES, $-1.96 ; P<0.00001)$, and anxiety (ES, -1.53 ; $P<0.00001$ ) [26] (Table 1).

The meta-analysis of electroacupuncture plus moxibustion versus oral SSRI showed a significant favorable effect of electroacupuncture plus moxibustion on outcome CAPS (2 studies, $n=115$, ES, -3.19 ; 95\% CI: -3.93 to $-2.46, P<$ 0.00001, heterogeneity: $\left.\chi^{2}=0.50, P=0.48, I^{2}=0 \%\right)$, 
TABLE 2: Cochrane risk of bias of included randomized controlled trials.

\begin{tabular}{|c|c|c|c|c|}
\hline $\begin{array}{l}\text { First author [ref] } \\
\text { (Year) }\end{array}$ & $\begin{array}{l}\text { Hollifield [18] } \\
\text { (2007) }\end{array}$ & Zhang [25] (2010) & $\begin{array}{c}\text { Zhang [26] } \\
(2010)\end{array}$ & $\begin{array}{c}\text { Zhang [27] } \\
\text { (2011) }\end{array}$ \\
\hline $\begin{array}{l}\text { (1) Random sequence generation } \\
\text { (selection bias) }\end{array}$ & $\begin{array}{c}\mathrm{L} \\
\text { (computerized randomization) }\end{array}$ & $\begin{array}{c}\mathrm{L} \\
\text { (computerized randomization) }\end{array}$ & $\mathrm{U}$ & $\mathrm{U}$ \\
\hline $\begin{array}{l}\text { (2) Allocation concealment } \\
\text { (selection bias) }\end{array}$ & $\begin{array}{c}\mathrm{L} \\
\text { (central allocation) }\end{array}$ & $\begin{array}{c}\mathrm{L} \\
\text { (sequentially numbered, opaque } \\
\text { sealed envelopes) }\end{array}$ & $\mathrm{U}$ & $\mathrm{U}$ \\
\hline $\begin{array}{l}\text { (3) Blinding of participants } \\
\text { (performance bias) }\end{array}$ & $\mathrm{H}$ & $\mathrm{H}$ & $\mathrm{H}$ & $\mathrm{H}$ \\
\hline $\begin{array}{l}\text { (4) Blinding of outcome assessment } \\
\text { (detection bias) }\end{array}$ & $\begin{array}{c}\mathrm{L} \\
\text { (mentioned) }\end{array}$ & $\begin{array}{c}\mathrm{L} \\
\text { (mentioned) }\end{array}$ & $\mathrm{U}$ & $\mathrm{U}$ \\
\hline $\begin{array}{l}\text { (5) Incomplete outcome data } \\
\text { (attrition bias) }\end{array}$ & $\begin{array}{c}\mathrm{L} \\
\text { (mentioned) }\end{array}$ & $\mathrm{U}$ & $\mathrm{U}$ & $\mathrm{U}$ \\
\hline $\begin{array}{l}\text { (6) Selective reporting } \\
\text { (reporting bias) }\end{array}$ & $\mathrm{U}$ & $\mathrm{U}$ & $\mathrm{U}$ & $\mathrm{U}$ \\
\hline $\begin{array}{l}\text { (7) Other sources of bias } \\
\text { (other bias) }\end{array}$ & $\mathrm{U}$ & $\mathrm{U}$ & $\mathrm{U}$ & $\mathrm{U}$ \\
\hline
\end{tabular}

L: low risk of bias; H: high risk of bias; U: unclear.

depression (2 studies, $n=115$, ES, -1.76 ; 95\% CI: -2.21 to $-1.31, P<0.00001$, heterogeneity: $\chi^{2}=1.04, P=0.31, I^{2}=$ $4 \%$ ), and anxiety ( 2 studies, $n=115$, ES, -1.14 ; 95\% CI: -1.44 to $-0.84, P<0.00001$, heterogeneity: $\chi^{2}=0.62, P=0.43, I^{2}$ $=0 \%)($ Table 4$)$.

3.3.5. Acupuncture Treatment in 2 UCTs. Two UCTs evaluated acupuncture treatment for total 103 earthquake-caused PTSD patients and showed effectiveness of 94.2\% [28] and 91.2\% [29], respectively (Table 1).

3.3.6. Adverse Events. Of all 6 studies, 2 RCTs described adverse events related to needle acupuncture [18, 25]. One study noted that some patients (original paper did not report the exact number) mentioned roughness of operational practices, fear of needles, bleeding, hematoma, pain, and fainting [25]. Another study reported just one perceived adverse effect (kidney pain) as a reason for withdrawal from acupuncture treatment [18]. No serious adverse events were reported.

\subsection{Risk of Bias and Reporting Quality}

3.4.1. Risk of Bias in Included RCTs Based on Cochrane Criteria. The risk of bias was low in one RCT [18], whereas one trial [25] had a moderate risk of bias and two trials $[26,27]$ had a high risk of bias in most categories (Table 2). Two RCTs employed adequate sequence generation methods and allocation concealment $[18,25]$, whereas the other two $[26,27]$ failed to report those categories. Assessor blinding was reported in the former two RCTs $[18,25]$. The risk of bias for incomplete outcome data was low in only one RCT [18]. In all, the four included RCTs had an unclear risk of bias in terms of selective reporting and other sources of bias. major international editorial groups have endorsed the CONSORT statement, and the statement facilitates critical appraisal and interpretation of RCTs [23]. For this reason, the current review assessed the reporting quality of included RCTs based on the CONSORT 2010 guideline. The 4 included RCTs described 22 items (59.5\%) [18] among 37 items, 15 items (40.5\%) [25], 9 items (24.3\%) [26], and 8 items (21.6\%) [27] according to the CONSORT 2010 checklist [23].

3.4.3. Reporting Quality of 4 Included RCTs Based on Revised STRICTA. The STRICTA reporting guideline is an extension of CONSORT was designed to improve the completeness and transparency of reporting of interventions in controlled trials of acupuncture [24], so that such trials may be more accurately interpreted and readily replicated [24]. The reporting quality of acupuncture was high for two of the included RCTs $[18,25]$, medium in one [26], and low in remaining RCT [27]. The 4 included RCTs reported 16 of 17 items (94.1\%) [18], 15 items (88.2\%) [25], 13 items (76.5\%) [26], and 8 items (47.1\%) [27] according to the revised STRICTA guideline. The two high-quality trials $[18,25]$ presented almost all items transparently except one or two items, whereas the lowquality trial [27] did not describe clearly even the reported 8 items (Table 3 ).

\section{Discussion}

This is the first systematic review and meta-analysis of prospective clinical trials on the effectiveness of acupuncture for treatment of PTSD. Only 4 RCTs and 2 UCTs met the inclusion criteria for this review. Our main finding of this review is that acupuncture is effective for PTSD based on one high-quality RCT [18] and a meta-analysis.

The high-quality RCT showed that acupuncture had statistically significant effects compared to a waitlist control, although no statistical difference was found between 
TABLE 3: Reporting quality of 4 included RCTs based on revised STRICTA.

\begin{tabular}{|c|c|c|c|c|}
\hline Checklist item & $\begin{array}{l}\text { Hollifield et al. [18] } \\
\text { (2007) }\end{array}$ & Zhang et al. [25] (2010) & Zhang et al. [26] (2010) & $\begin{array}{l}\text { Zhang et al. [27] } \\
\text { (2011) }\end{array}$ \\
\hline \multicolumn{5}{|l|}{ (1) Acupuncture rationale } \\
\hline $\begin{array}{l}\text { (1a) Style of } \\
\text { acupuncture }\end{array}$ & TCM & TCM & TCM & n.r. \\
\hline $\begin{array}{l}\text { (1b) Reasoning for } \\
\text { treatment provided }\end{array}$ & $\begin{array}{l}\text { A paper by Napadow et al., } \\
2005[42]\end{array}$ & $\begin{array}{l}\text { A paper by Hollifield et al., } \\
2007[18]\end{array}$ & $\begin{array}{l}\text { A paper by Hollifield et al., } \\
2007[18]\end{array}$ & n.r. \\
\hline $\begin{array}{l}\text { (1c) Extent to which } \\
\text { treatment was varied }\end{array}$ & $\begin{array}{l}2 \text { types of AT } \\
\text { (1) AT: } 25 \text { fixed needles plus } \\
\text { up to } 3 \text { flexible needles } \\
\text { within } 15 \text { points } \\
\text { (2) AAT: } \geq 6 \text { vaccaria seeds }\end{array}$ & $\begin{array}{l}\text { Fixed interventions } \\
\text { (A) EA only } \\
\text { (B) EA + moxa } \\
\text { (C) EA + AAT }\end{array}$ & $\begin{array}{l}\text { Fixed interventions } \\
\text { EA + moxa }\end{array}$ & Fixed intervention \\
\hline
\end{tabular}

(2) Details of needling

(2a) Number of needle insertions

per subject per session used

(2b) Names of points

(1) AT: 25 plus up to 3 needles

(2) AAT: $\geq 6$ vaccaria seeds

(1) EA: 8 needles

(2) AAT: 6 vaccaria seeds

EA: 8 needles

unclear.

(1) EA: bilateral at

(1) AT: bilateral at LR3, GB20/unilateral at GV24, PC6, HT7, ST36, SP6, $\quad$ EX-HN1, GV20 GB20, BL14, 15, 18, 20, 21 (2) AAT: unilateral at and 23/unilateral at Yintang Subcortex, Shenmen, (2) AAT: unilateral at Sympathetic, Heart, Liver, Shenmen, Sympathetic, Kidney Liver, Kidney, Lung points

(3) moxa: bilateral at BL23, BL52/unilateral at GV4
(2c) Depth of insertion
(1) AT: $1 / 4$ to $1 / 2$ inch
(2) AAT: not inserted
(1) AT: n.r.
(2d) Responses sought
(2) AAT: not applicable
(1) AT: manipulation
(2e) Needle stimulation (2) AAT: self-massage on

(1) EA: 0.5 to 1.2 cun
(2) AAT: not inserted

(1) EA: de-qi

(2) AAT: not applicable.

(1) EA: electrical the seeds for $15 \mathrm{~min} / \mathrm{d}$

(2f) Needle retention
time

(1) AT: $25-40 \mathrm{~min}$

(2) AAT: unclear

(2g) Needle type

(1) AT: Viva needles, $34 \mathrm{~g}$

(2) AAT: vaccaria seeds stimulation, $100 \mathrm{~Hz}$

(2) AAT: 1-2 min pressure

(A) $30 \mathrm{~min}$

(B) $30 \mathrm{~min}$

(C) $30 \mathrm{~min}$

(1) EA: $0.30 \mathrm{~mm} \times 40 \mathrm{~mm}$

(2) AAT: vaccaria seeds
EX-HN1, GV20
(1) EA: bilateral at

GB20/unilateral at GV24,

Unilateral at left PC8

(2) moxa: bilateral at BL23,

BL52/unilateral at GV4

EA: 0.5 to 1.2 cun

n.r.

EA: de-qi

n.r.

EA: electrical stimulation, $5 \sim 8.3 \mathrm{~Hz}$

$30 \mathrm{~min}$

n.r.
A Japanese stimulator with $50 \mathrm{~Hz}$ was used

Unclear, but the left PC8 was stimulated for $30 \mathrm{~min}$

n.r.

(3) Treatment regimen

(3a) Number of treatment sessions

(3b) Frequency and duration of treatment sessions
24 sessions $\quad 36$ sessions

Twice a week, 1 hour per session, 12 weeks
Three times a week, 12 weeks
(1) EA: 18 sessions

(2) moxa: 36 sessions

3 4 sessions*

(1) EA: three times a week, 6 weeks

(2) moxa: three times a week, 12 weeks

(4) Other components

of treatment

(4a) Details of other interventions administered to the acupuncture group

(4b) Setting and contex of treatment
Patients were taught how to use vaccaria seeds for symptom management
(3) moxa: $30 \mathrm{~g}$ and $20 \mathrm{~min} / \mathrm{session}$, wooden moxibustion box $20 \mathrm{~mm} \times$ $15 \mathrm{~mm} \times 12 \mathrm{~mm}$
(2) moxa: $20 \mathrm{~min} / \mathrm{session} \quad \mathrm{CBT}$

A time every other day for 1 week

n.r.

n.r. 
TABle 3: Continued.

\begin{tabular}{lllll}
\hline Checklist item & $\begin{array}{l}\text { Hollifield et al. [18] } \\
(2007)\end{array}$ & Zhang et al. [25] (2010) & Zhang et al. [26] (2010) & $\begin{array}{l}\text { Zhang et al. [27] } \\
(2011)\end{array}$ \\
\hline $\begin{array}{l}\text { (5) Practitioner } \\
\text { background }\end{array}$ & $\begin{array}{l}\text { Doctor of Oriental } \\
\text { Medicine in New Mexico }\end{array}$ & & & \\
$\begin{array}{l}\text { (5) Description of } \\
\text { participating } \\
\text { Acupuncturists }\end{array}$ & $\begin{array}{l}\text { with 4 years postgraduate } \\
\text { TCM clinical experience }\end{array}$ & & n.r. & n.r. \\
\hline
\end{tabular}

(6) Control or comparator interventions

(6a) Rationale for the control or comparator

(6b) Precise description of the control or comparator
(B) A review by Bisson and Andrew, 2005 [43]

(C) not applicable

(B) $\mathrm{CBT}$

(C) WLC
Approval of FDA

(D) Oral SSRI (Paroxetine

$20 \mathrm{mg}$, once/day, 12 weeks) n.r.

n.r.

Oral SSRI (Paroxetine $20 \mathrm{mg}$, once/day, 12 weeks)
(B) $\mathrm{CBT}$

Abbreviations: RCT: randomized controlled trial; TCM: traditional Chinese medicine; n.r: not reported; AT: classical acupuncture; EA: electro-acupuncture; moxa, moxibustion; AAT: auricular acupuncture; CBT: cognitive behavioral therapy; WLC: waitlist control; SSRI: selective serotonin reuptake inhibitors.

${ }^{*}$ treated a time every other day for 1 week.

TABLE 4: Meta-analysis of acupuncture for posttraumatic stress disorder. PTSD: posttraumatic stress disorder; CAPS, clinician-administered PTSD scale; HAMD, Hamilton depression rating scale; HAMA, Hamilton anxiety rating scale; EA, electro-acupuncture; moxa, moxibustion; SSRI, selective serotonin reuptake inhibitors;

(a) PTSD scale (CAPS)

\begin{tabular}{|c|c|c|c|c|c|c|c|c|c|}
\hline \multirow{2}{*}{$\begin{array}{l}\text { Study or } \\
\text { subgroup }\end{array}$} & \multicolumn{3}{|c|}{ EA + Moxa } & \multicolumn{3}{|c|}{ SSRI } & \multicolumn{2}{|r|}{ Mean difference } & Mean difference \\
\hline & Mean & SD & Total & Mean & SD & Total & Weight & IV, random, 95\% CI & IV, random, 95\% CI \\
\hline $\begin{array}{l}\text { Zhang et al., } 2010 \\
{[25]}\end{array}$ & -36.15 & 21.72 & 69 & -35.58 & 22.12 & 69 & $1.0 \%$ & $-0.57[-7.88,6.74]$ & \\
\hline $\begin{array}{l}\text { Zhang et al., } 2010 \\
{[26]}\end{array}$ & -17.17 & 1.84 & 46 & -13.95 & 1.76 & 46 & $99.0 \%$ & $-3.22[-3.96,-2.48]$ & \\
\hline $\begin{array}{l}\text { Total }(95 \% \mathrm{CI}) \\
\text { Heterogeneity: } \tau^{2} \\
\text { Test for overall eff }\end{array}$ & $\begin{array}{l}=0.00, \chi \\
\text { et: } Z=8\end{array}$ & $\begin{array}{l}=0.50 \\
5(P\end{array}$ & $\begin{array}{l}115 \\
\mathrm{df}=1 \\
0.000\end{array}$ & $P=0.48)$ & $I^{2}=0$ & 115 & $100.0 \%$ & $-3.19[-3.93,-2.46]$ & 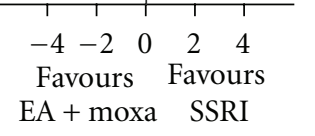 \\
\hline
\end{tabular}

\begin{tabular}{|c|c|c|c|c|c|c|c|c|c|}
\hline \multirow{2}{*}{$\begin{array}{l}\text { Study or } \\
\text { subgroup }\end{array}$} & \multicolumn{3}{|c|}{ EA + Moxa } & \multicolumn{3}{|c|}{ SSRI } & \multirow[b]{2}{*}{ Weight } & \multirow{2}{*}{$\begin{array}{c}\text { Mean difference } \\
\text { IV, random, 95\% CI }\end{array}$} & \multirow{2}{*}{$\begin{array}{c}\text { Mean difference } \\
\text { IV, random, 95\% CI }\end{array}$} \\
\hline & Mean & $\mathrm{SD}$ & Total & Mean & SD & Total & & & \\
\hline $\begin{array}{l}\text { Zhang et al., } 2010 \\
{[25]}\end{array}$ & -7.42 & 5.58 & 69 & -6.55 & 5.1 & 69 & $6.2 \%$ & $-0.87[-2.65,0.91]$ & \\
\hline $\begin{array}{l}\text { Zhang et al., } 2010 \\
\text { [26] }\end{array}$ & -7.27 & 0.93 & 46 & -5.45 & 0.91 & 46 & $93.8 \%$ & $-1.82[-2.20,-1.44]$ & \\
\hline & & & 115 & & & 115 & $100.0 \%$ & $-1.76[-2.21,-1.31]$ & $\begin{array}{ccccc} & 1 & & 1 & 1 \\
-4 & -2 & 0 & 2 & 4\end{array}$ \\
\hline \multicolumn{9}{|c|}{ Heterogeneity: $\tau^{2}=0.02, \chi^{2}=1.04, \mathrm{df}=1(P=0.31) ; I^{2}=4 \%$} & $\begin{array}{cc}\text { Favours } & \text { Favours } \\
\text { EA + moxa } & \text { SSRI }\end{array}$ \\
\hline
\end{tabular}

Test for overall effect: $Z=7.72(P<0.00001)$

(c) Anxiety (HAMA).

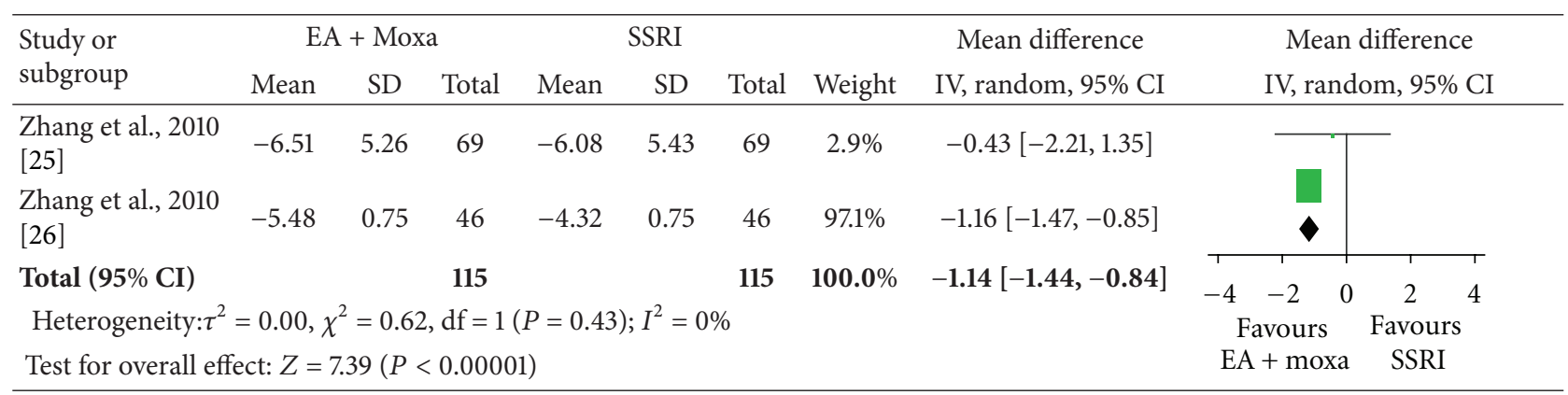


acupuncture and CBT. Also the therapeutic effect of acupuncture was similar with CBT therapy based on the trial. Additionally, the clinical improvement related to acupuncture or CBT lasted for at least 3 months after the end of treatment in the high-quality RCT.

The meta-analysis showed that acupuncture plus moxibustion was superior to oral SSRI for PTSD. But, we should interpret these results with caution because the meta-analysis was based on one medium-quality RCT [25] and one lowquality RCT [26].

One RCT [27] showed that acupoint stimulation plus CBT was more effective than CBT alone in reducing PTSD symptoms. However, acupuncture treatment was not described transparently. Therefore, this result had doubtful reliability.

We found a similar pattern of reporting quality when comparing the Cochrane risk of bias [22] with the CONSORT 2010 checklist [23]. Two of the included studies [18, 25] had a high reporting quality in terms of acupuncture based on the revised STRICTA guideline [24]. All the studies failed to describe in detail adverse effects related to acupuncture.

We would like to emphasize the clinical importance of acupuncture for PTSD. Acupuncture might be useful in emergency medicine [44]. A recent case series study suggested possible effectiveness of acupuncture in emergency conditions involving PTSD and emotional trauma [45]. In addition, acupuncture is a conveniently portable medical device for taking emergency measures, and it is very cheap, safe, and easy to handle for trained practitioners.

According to a study [46], during long-term SSRI therapy, the most troubling adverse effects were sexual dysfunction, weight gain, and sleep disturbance. The incidence rate of sexual dysfunction was reported as $2 \%$ to $7 \%$ [47]. Mean weight gain of $10.8 \mathrm{~kg}$ (24lbs) was found after 6 to 12 months of paroxetine therapy [48]. On the other hand, for acupuncture, "mild" adverse events of such as bleeding, bruising, pain on needling occurred in rate of $6.8 \%$ (2,178 out of 31,822 sessions) [49]. And no serious adverse events were reported in total 66,229 treatment sessions according to two studies $[49,50]$. Therefore acupuncture may be a relatively safe alternative for PTSD in contrast to SSRI, if long-term therapy is needed for treatment.

This systematic review has several limitations. First, although we made strong efforts to retrieve all RCTs on the subject, the evidence reviewed is potentially incomplete because only one rigorous study was included. Second, because there was no RCT on PTSD with a sham acupuncture control, we could not evaluate the effects of acupuncture compared to an inert placebo control [51]. Third, study design was quite different across the four included RCTs. Two RCTs $[18,25]$ compared acupuncture with different controls (CBT and oral SSRI), and the other two RCTs [26, 27] employed acupuncture as a cointervention of moxibustion and CBT. These very different designs across studies prevented us from abstracting a firm conclusion. Furthermore, the paucity of included trials and the suboptimal methodological quality of the primary data overall, except for one high-quality trial, are also important vulnerabilities of this review.
In total, from these drawbacks we could suggest several important recommendations for future research in this area. One is a need for appropriate controls such as sham/placebo control or other relevant active controls for testing the efficacy or effectiveness of acupuncture for PTSD in the design of parallel RCT or comparative effectiveness research. The second is outcomes should be used by validated one as primary one is PTSD scale and the secondary one is depression or anxiety with safety reporting. The third is high methodological quality is strongly required, as adequate randomization with allocation concealment, blinding of participants and assessors, or sample size estimation for power of trial, with following guideline of CONSORT and STRICTA.

\section{Conclusions}

The results of this systematic review and meta-analysis suggest that evidence of the effectiveness of acupuncture for PTSD is encouraging but not cogent, because only two RCTs were included in meta-analysis, and it is too small to verify the efficacy of acupuncture. For the future researches, shamcontrolled RCTs [52] or comparative effectiveness researches [53] are required to test efficacy and effectiveness of acupuncture for PTSD. To prevent performance bias and detection bias, blinding of participants and outcome assessment should be kept in future trials, too.

\section{Disclosure}

The authors report no financial relationship or other relevant to the subject of this paper.

\section{References}

[1] R. V. Reed, M. Fazel, and L. Goldring, "Post-traumatic stress disorder," British Medical Journal, vol. 344, Article ID e3790, 2012.

[2] A. Y. Shalev, "What is posttraumatic stress disorder?" Journal of Clinical Psychiatry, vol. 62, supplement 17, pp. 4-10, 2001.

[3] R. A. Bryant, "Acute stress disorder as a predictor of posttraumatic stress disorder: a systematic review," Journal of Clinical Psychiatry, vol. 72, no. 2, pp. 233-239, 2011.

[4] National Collaborating Centre for Mental Health (UK), PostTraumatic Stress Disorder: The Management of PTSD in Adults and Children in Primary and Secondary Care, Gaskell and British Psychological Society, 2005.

[5] Australian Centre for Posttraumatic Mental Health, "Evidence review and treatment recommendations for adults with PTSD," in Australian Guidelines for the Treatment of Adults with Acute Stress Disorder and Posttraumatic Stress Disorder, pp. 33-101, Australian Centre for Posttraumatic Mental Health, 2007.

[6] J. L. Strauss, R. Coeytaux, J. McDuffie, A. Nagi, and J. Williams, Efficacy of Complementary and Alternative Medicine Therapies for Posttraumatic Stress Disorder, VA Evidence-based Synthesis Program Reports, Washington, DC, USA, 2011.

[7] J. Unutzer, R. Klap, R. Sturm et al., "Mental disorders and the use of alternative medicine: results from a national survey," American Journal of Psychiatry, vol. 157, no. 11, pp. 1851-1857, 2000 . 
[8] L. S. Nasir, "Acupuncture," Primary Care, vol. 29, no. 2, pp. $393-$ 405, 2002.

[9] L. Conboy, M. St John, and R. Schnyer, "The effectiveness of acupuncture in the treatment of Gulf War Illness," Contemporary Clinical Trials, vol. 33, pp. 557-562, 2012.

[10] K. Pilkington, G. Kirkwood, H. Rampes, M. Cummings, and J. Richardson, "Acupuncture for anxiety and anxiety disorders-a systematic literature review," Acupuncture in Medicine, vol. 25, no. 1-2, pp. 1-10, 2007.

[11] M. S. Lee, B. C. Shin, and E. Ernst, "Acupuncture for Alzheimer's disease: a systematic review," International Journal of Clinical Practice, vol. 63, no. 6, pp. 874-879, 2009.

[12] S. Fogarty, D. Harris, C. Zaslawski, A. J. McAinch, and L. Stojanovska, "Acupuncture as an adjunct therapy in the treatment of eating disorders: a randomised cross-over pilot study," Complementary Therapies in Medicine, vol. 18, no. 6, pp. 233240, 2010.

[13] P. Ronan, N. Robinson, D. Harbinson, and D. MacInnes, "A case study exploration of the value of acupuncture as an adjunct treatment for patients diagnosed with schizophrenia: results and future study design," Journal of Chinese Integrative Medicine, vol. 9, no. 5, pp. 503-514, 2011.

[14] C. Becker-Carus, T. Heyden, and A. Kelle, "Effectiveness of acupuncture and attitude-relaxation training for treatment of primary sleep disorders," Zeitschrift fur Klinische Psychologie, Psychopathologie und Psychotherapie, vol. 33, no. 2, pp. 161-172, 1985.

[15] J.-G. Lin, Y.-Y. Chan, and Y.-H. Chen, "Acupuncture for the treatment of opiate addiction," Evidence-Based Complementary and Alternative Medicine, vol. 2012, Article ID 739045, 10 pages, 2012.

[16] C. H. Yang, B. H. Lee, and S. H. Sohn, "A possible mechanism underlying the effectiveness of acupuncture in the treatment of drug addiction," Evidence-based Complementary and Alternative Medicine, vol. 5, no. 3, pp. 257-266, 2008.

[17] L. Eshkevari, R. Egan, D. Phillips et al., "Acupuncture at ST36 prevents chronic stress-induced increases in neuropeptide $\mathrm{Y}$ in rat," Experimental Biology and Medicine, vol. 237, pp. 18-23, 2012.

[18] M. Hollifield, N. Sinclair-Lian, T. D. Warner, and R. Hammerschlag, "Acupuncture for posttraumatic stress disorder: a randomized controlled pilot trial," Journal of Nervous and Mental Disease, vol. 195, no. 6, pp. 504-513, 2007.

[19] D. Feinstein, "Rapid treatment of PTSD: why psychological exposure with acupoint tapping may be effective," Psychotherapy, vol. 47, no. 3, pp. 385-402, 2010.

[20] M. Hollifield, "Acupuncture for posttraumatic stress disorder: conceptual, clinical, and biological data support further research," CNS Neuroscience and Therapeutics, vol. 17, pp. 769779, 2011.

[21] S. Bidwell and M. Jensen, "Using a search protocol to identify sources of information: the COSI Model," in Etext on Health Technology Assessment (HTA) Information Resources, Chapter 3, http://www.nlm.nih.gov/archive/20060905/nichsr/ehta/chapter3.html\#1.

[22] J. Higgins and S. Green, Cochrane Handbook for Systematic Reviews of Interventions, Version 5. 1. 0., 2011.

[23] D. Moher, S. Hopewell, K. F. Schulz et al., "CONSORT 2010 explanation and elaboration: updated guidelines for reporting parallel group randomised trials," Journal of Clinical Epidemiology, vol. 63, no. 8, pp. e1-e37, 2010.
[24] H. MacPherson, D. G. Altman, R. Hammerschlag et al., "Revised standards for reporting interventions in clinical trials of acupuncture (STRICTA): extending the consort statement," Acupuncture in Medicine, vol. 28, no. 2, pp. 83-93, 2010.

[25] H. Zhang, C. Yuan, L. Ran et al., "RCT research of different acupuncture therapies in treating Posttraumatic stress disorder after Wenchuan "5.12" earthquake," China Journal of Traditional Chinese Medicine and Pharmacy, vol. 25, pp. 1505-1510, 2010 (Chinese).

[26] H. Zhang, L. Ran, X. Yuan, K. Wang, Z. Hu, and J. Yang, "Clinical observation on acupuncture and moxibustion in treating post traumatic stress disorder after 5.12 earthquake," Journal of Chengdu University of TCM, vol. 33, article 4, 2010.

[27] Y. Zhang, B. Feng, J. P. Xie, F. Z. Xu, and J. Chen, "Clinical study on treatment of the earthquake-caused post-traumatic stress disorder by cognitive-behavior therapy and acupoint stimulation," Journal of Traditional Chinese Medicine, vol. 31, no. 1, pp. 60-63, 2011.

[28] Y. Wang and Y. Hu, "Acupuncture and moxibustion treatment for 69 cases of posttraumatic stress disorder caused by an earthquake," Henan Traditional Chinese Medicine, vol. 29, article 291, 2009 (Chinese).

[29] X. Yuan, C. Liu, and R. Lai, "Acupuncture treatment for 34 cases of posttraumatic stress disorder," Zhongguo Zhen Jiu, vol. 29, article 234, 2009 (Chinese).

[30] H. Zhang, W. Chen, W. Song, M. Zhu, and Y. Feng, "Brain glucose metabolism involved in acupuncture treatment on posttraumatic stress disorder patients," China Journal of Traditional Chinese Medicine and Pharmacy, vol. 25, pp. 1882-1884, 2010 (Chinese).

[31] M. K. Prisco, "Examining the Effect of Acupuncture on Sleep Difficulties Related to Post Traumatic Stress Disorder," http://www.clinicaltrials.gov/ct2/show/NCT00868517.

[32] T. W. Findley, "Pilot of Acupuncture to Improve Quality of Life in Veterans With Traumatic Brain Injury (TBI) and PostTraumatic Stress Disorder (PTSD)," http://www.clinicaltrials.gov/ct2/show/NCT01060553.

[33] Acupuncture for the Treatment of Post-Traumatic Stress Disorder (PTSD), http://www.clinicaltrials.gov/ct2/show/NCT00055354.

[34] N. Money, M. Fritts, R. Miller, K. Gore, and C. Engel, Integrative Restoration as an Adjunctive Therapy for Post-Traumatic Stress Disorder, The Uniformed Services University of the Health Sciences Research Week, Bethesda, MD, USA, 2009.

[35] Y. Wang and Y. Hu, "Electro-acupuncture on head points in treating posttraumatic stress disorder after "5.12" earthquake: a randomized controlled trial," in Acupuncture, Moxibustion and Tuina, Chengdu University of TCM, Chengdu, China, 2010.

[36] H. Zhang, X. Yuan, L. Ran et al., "Electro-acupuncture on head points in treating posttraumatic stress disorder: a randomized controlled trial and PET imaging observation," in Proceedings of the 5th Psychosomatic Medicine Symposium of National Integrative Medicine, Tianjin, China, 2010.

[37] Z. Hu and H. Zhang, "Electro-acupuncture on head points and moxibustion in treating posttraumatic stress disorder after Wenchuan "5.12: earthquake: a randomized controlled trial," in Acupuncture, Moxibustion and Tuina, Chengdu University of TCM, Chengdu, China, 2010.

[38] K. Wang and H. Zhang, "Electro-acupuncture on head points and auricular acupuncture in treating posttraumatic stress disorder after "5.12" earthquake: a randomized controlled trial," 
in Acupuncture, Moxibustion and Tuina, Chengdu University of TCM, Chengdu, China, 2010.

[39] Y. Wang, Y. Hu, W. Wang, R. Pang, and A. Zhang, "Clinical studies on treatment of earthquake-caused posttraumatic stress disorder using electro-acupuncture," Evidence-Based Complementary and Alternative Medicine, vol. 2012, Article ID 431279, 7 pages, 2012.

[40] A. Liberati, D. G. Altman, J. Tetzlaff et al., "The PRISMA statement for reporting systematic reviews and meta-analyses of studies that evaluate health care interventions: explanation and elaboration," PLoS Medicine, vol. 6, no. 7, Article ID e1000100, 2009.

[41] J. Cohen, Statistical Power Analysis for the Behavioral Sciences, Lawrence Erlbaum Associate, New Jersey, NJ, USA, 2nd edition, 1988.

[42] V. Napadow, N. Makris, J. Liu, N. W. Kettner, K. K. Kwong, and K. K. S. Hui, "Effects of electroacupuncture versus manual acupuncture on the human brain as measured by fMRI," Human Brain Mapping, vol. 24, no. 3, pp. 193-205, 2005.

[43] J. Bisson and M. Andrew, "Psychological treatment of posttraumatic stress disorder (PTSD)," Cochrane Database of Systematic Reviews, no. 2, p. CD003388, 2005.

[44] C. H. Hsu, Y. Hua, G. P. Jong et al., "Shock resuscitation with acupuncture: case report," Emergency Medicine Journal, vol. 23, no. 3, p. e18, 2006.

[45] J. Fleckenstein, J. Schottdorf, U. Kreimeier, and D. Irnich, "Acupuncture in emergency medicine: results of a case series," Der Anaesthesist, vol. 60, pp. 854-862, 2011.

[46] D. P. Sniezek, "Guest editorial: community-based wounded warrior sustainability initiative (CBWSI): an integrative medicine strategy for mitigating the effects of PTSD," Journal of Rehabilitation Research and Development, vol. 49, pp. IX-XIX, 2012.

[47] A. I. Montejo, G. Llorca, J. A. Izquierdo et al., "Sexual dysfunction secondary to SSRIs. A comparative analysis in 308 patients," Actas Luso-Españolas de Neurología, Psiquiatría y Ciencias Afines, vol. 24, no. 6, pp. 311-321, 1996.

[48] N. Sussman and D. Ginsberg, "Rethinking side effects of the selective serotonin reuptake inhibitors: sexual dysfunction and weight gain," Psychiatric Annals, vol. 28, no. 2, pp. 89-97, 1998.

[49] A. White, S. Hayhoe, A. Hart, and E. Ernst, "Adverse events following acupuncture: prospective survey of 32000 consultations with doctors and physiotherapists," British Medical Journal, vol. 323, no. 7311, pp. 485-486, 2001.

[50] H. MacPherson, K. Thomas, S. Walters, and M. Fitter, “The York acupuncture safety study: prospective survey of 34000 treatments by traditional acupuncturists," British Medical Journal, vol. 323, no. 7311, pp. 486-487, 2001.

[51] J. Park, A. White, C. Stevinson, E. Ernst, and M. James, "Validating a new non-penetrating sham acupuncture device: two randomised controlled trials," Acupuncture in Medicine, vol. 20, no. 4, pp. 168-174, 2002.

[52] B. V. Watts, B. Landon, A. Groft, and Y. Young-Xu, "A sham controlled study of repetitive transcranial magnetic stimulation for posttraumatic stress disorder," Brain Stimulation, vol. 5, pp. 38-43, 2012.

[53] R. M. Golub and P. B. Fontanarosa, "Comparative effectiveness research: relative successes," The Journal of the American Medical Association, vol. 307, pp. 1643-1645, 2012. 


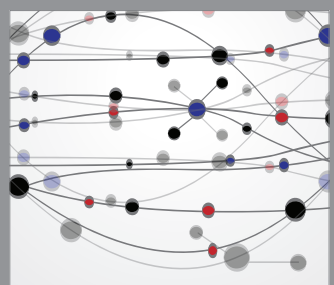

The Scientific World Journal
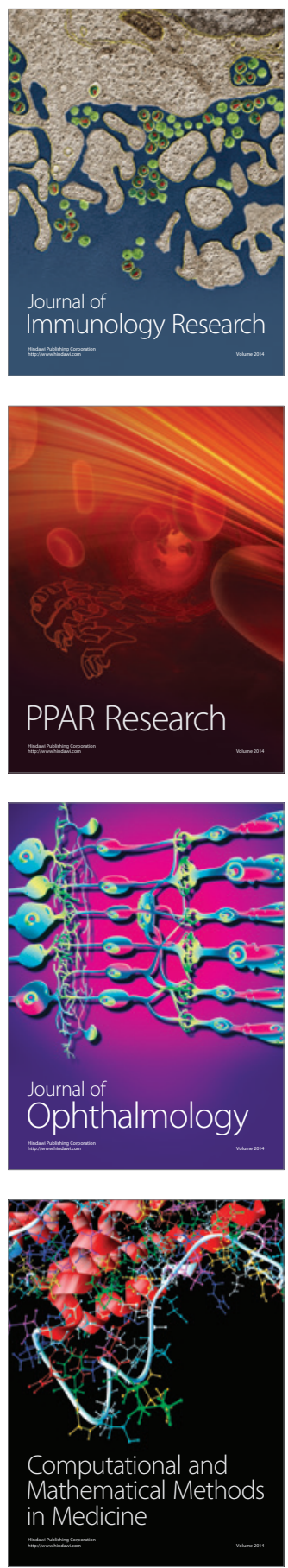

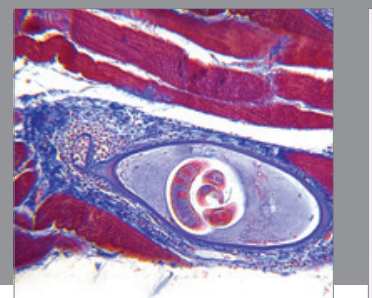

Gastroenterology

Research and Practice
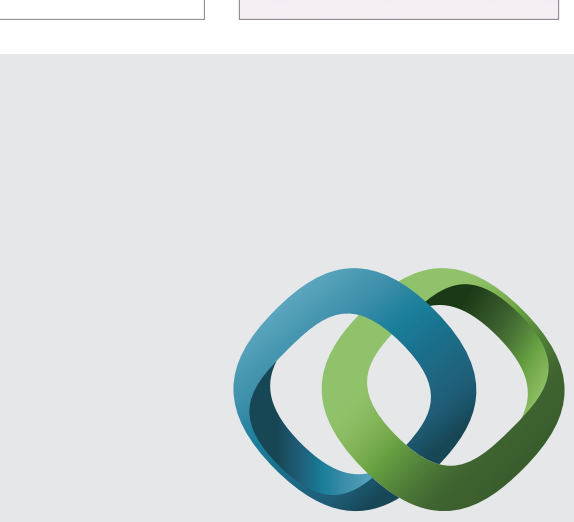

\section{Hindawi}

Submit your manuscripts at

http://www.hindawi.com
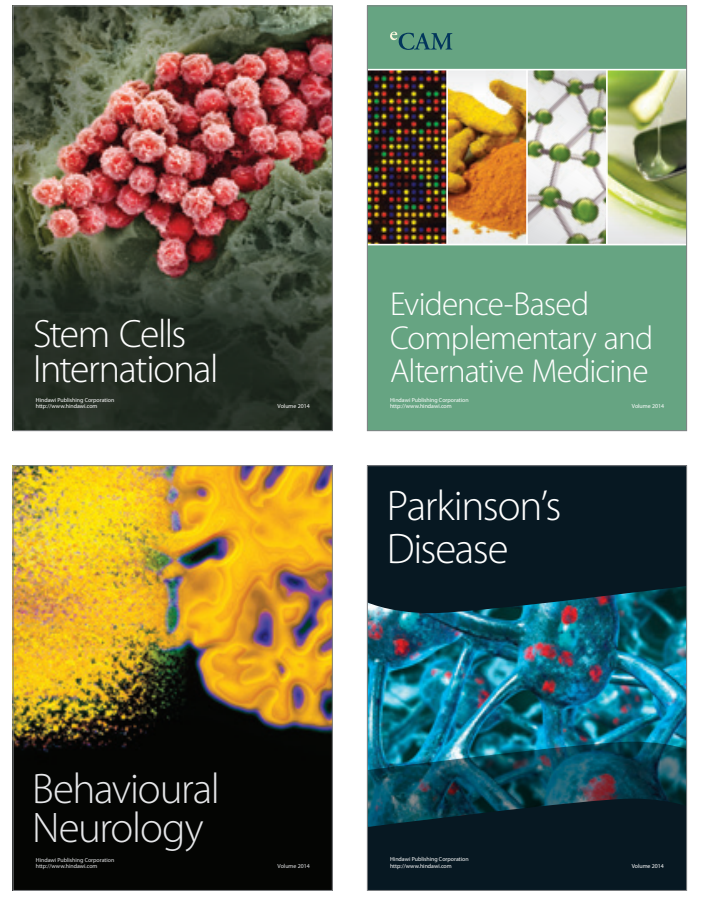
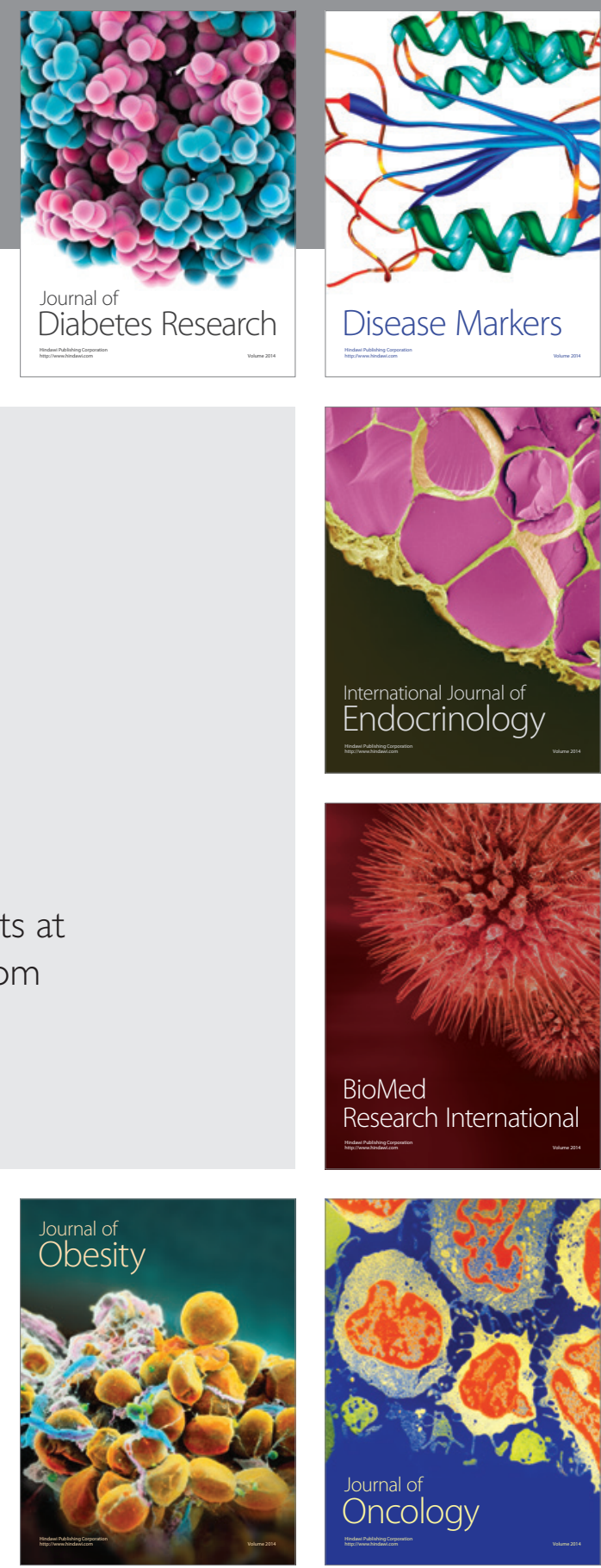

Disease Markers
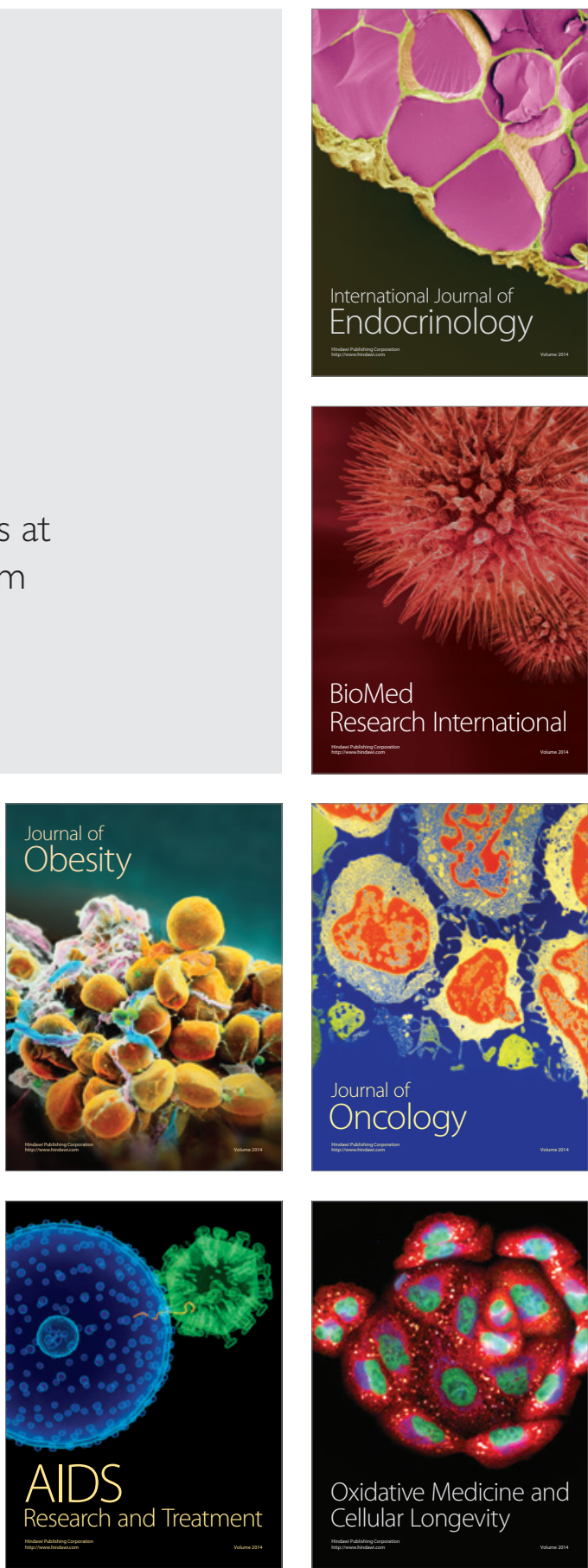